\title{
Correction to: On the Applicability of the Expected Waiting Time Method in Nonstationary Flood Design
}

\section{Lei Yan ${ }^{1} \cdot$ Lihua Xiong $^{2} \cdot$ Qinghua Luan ${ }^{1} \cdot$ Cong Jiang ${ }^{3} \cdot$ Kunxia $\mathrm{Yu}^{4} \cdot$ Chong-Yu Xu $^{5}$}

Published online: 1 July 2020

(C) Springer Nature B.V. 2020

\section{Correction to: Water Resour Manag \\ https://doi.org/10.1007/s11269-020-02581-w}

The original version of this article unfortunately contains mistakes in equations 1 and 2 . The mistakes and corrections is described below:

The online version of the original article can be found at https://doi.org/10.1007/s11269-020-02581-w

Qinghua Luan

caro197011202@163.com

Lei Yan

yan1@whu.edu.cn

Lihua Xiong

xionglh@whu.edu.cn

Cong Jiang

jiangcong@cug.edu.cn

Kunxia $\mathrm{Yu}$

yukunxia@126.com

Chong-Yu Xu

c.y.xu@geo.uio.no

1 College of Water Conservancy and Hydropower, Hebei University of Engineering, Handan 056021, People's Republic of China

2 State Key Laboratory of Water Resources and Hydropower Engineering Science, Wuhan University, Wuhan 430072, China

3 School of Environmental Studies, China University of Geosciences (Wuhan), Wuhan 430074, China

4 State Key Laboratory of Eco-hydraulics in Northwest Arid Region of China, Xi'an University of Technology, Xi'an 710048, China

5 Department of Geosciences, University of Oslo, P.O. Box 1022, Blindern, N-0315 Oslo, Norway 


$$
\begin{gathered}
f(x)=P(X=x)=p_{x} \prod_{t=1}^{x-1}\left(1-p_{t}\right) x=1,2, \ldots, x_{\max } \\
m=E(X)=\sum_{x=1}^{x_{\max }} x f(x)=\sum_{x=1}^{x_{\max }} x\left(1-G_{Z, t}\left(z^{E W T}(m) \mid \boldsymbol{\theta}_{x}\right)\right) \prod_{t=1}^{x-1} G_{Z, t}\left(z^{E W T}(m) \mid \boldsymbol{\theta}_{t}\right)
\end{gathered}
$$

Should be:

$$
\begin{gathered}
f(x)=P(X=x)=p_{x} \prod_{t=1}^{x-1}\left(1-p_{t}\right) \quad x=1,2, \ldots, x_{\max } \\
m=E(X)=\sum_{x=1}^{x_{\max }} x f(x)=\sum_{x=1}^{x_{\max }} x\left(1-G_{Z, x}\left(z^{E W T}(m) \mid \boldsymbol{\theta}_{x}\right)\right) \prod_{t=1}^{x-1} G_{Z, t}\left(z^{E W T}(m) \mid \boldsymbol{\theta}_{t}\right)
\end{gathered}
$$

Publisher's Note Springer Nature remains neutral with regard to jurisdictional claims in published maps and institutional affiliations. 